Portland State University

PDXScholar

\title{
Developmental Programming Effects of Maternal Obesity
}

Carolle J. Kassab

Portland State University

Follow this and additional works at: https://pdxscholar.library.pdx.edu/honorstheses

Part of the Biology Commons, Developmental Biology Commons, and the Nutrition Commons Let us know how access to this document benefits you.

\section{Recommended Citation}

Kassab, Carolle J., "Developmental Programming Effects of Maternal Obesity" (2020). University Honors Theses. Paper 850.

https://doi.org/10.15760/honors.870

This Thesis is brought to you for free and open access. It has been accepted for inclusion in University Honors Theses by an authorized administrator of PDXScholar. Please contact us if we can make this document more accessible: pdxscholar@pdx.edu. 


\section{Developmental Programming Effects of Maternal Obesity}

By

Carolle Kassab

An undergraduate honors thesis submitted in partial fulfillment of the requirements for the degree of

Bachelor of Science

in

University Honors

and

Biology and Science

Thesis Advisor

Alina Maloyan, Ph.D

Portland State University 


\begin{abstract}
Population studies within the United States indicate increasing rates of obesity, considerably prominent for women within reproductive age. Maternal obesity is associated with the offspring's hyperlipidemia, diabetes, and cardiovascular disease, which have chronic consequences. Obesity in pregnancy causes metabolic and epigenetic perturbations within the fetal environment, disrupting future health of offspring. This phenomenon is known as developmental programming. Although the relationship between maternal undernutrition and developmental programming has been demonstrated, its relationship to maternal obesity remains understudied.
\end{abstract}

\title{
Obesity
}

The obesity rates in the United States have increased from $30.5 \%$ in 1999 to $42.2 \%$ in 2018, while severe obesity increased from $4.7 \%$ to $9.2 \%$ (Hales et al., 2020). It is projected that by $2030,50 \%$ of adults will be obese (Youfa et al., 2019), while $25 \%$ of adults will be morbidly obese (Ward et al., 2019). These trends can be accounted for by increased food supply, consumption of high calorie diets or processed foods, and large portion sizes. Additionally, lifestyle changes such as a decrease in physical activity and increase in sedentary activities have become more prominent. Cultural changes show a heavier emphasis on spending time watching television and electronic devices. Thus, obesity becomes a major public health issue that needs to be addressed.

Obesity is a risk factor for chronic health conditions such as cardiovascular disease and diabetes, major causes of death in the U.S. (Youfa et al., 2020). Accumulation of adipose tissue around vital organs is the hallmark of obesity, which is a factor in developing chronic disease. One reason for this is that excess fat leads to mechanical stress and compression on organsaffecting their proper function. Increased pressure and resistance on arteries require the heart to work harder, which increases the risk for developing high blood pressure. If these symptoms persist long term, this causes hypertension in obese individuals. This is one of the reasons that secondary disease, which is often seen in the obese population, tends to develop. 
Additionally, the accumulation of adipose tissue increases production of cell-signaling molecules, such as adipocytokines, that promote a pro-inflammatory state throughout the body (Heymsfield et al., 2017). These adipocytokines include interleukin-(IL)-6, 8, and 12, and are secreted by macrophages and adipocytes within adipose tissue. Pro-inflammatory factors are directly linked to insulin resistance and progression of Type 2 Diabetes (Castro et al., 2017). This is caused by the infiltration of macrophages into locations of fat accumulation, where adipocytokines can be released. These molecules then interfere with insulin signaling, and contribute to insulin resistance, which is caused by the release of free fatty acids from excess fat accumulation (Castro et al., 2017).

Pro-inflammatory factors are further promoted by another adipokine, Leptin, which recruits more macrophages for the secretion of these molecules. Leptin resistance is common within obese individuals, and interferes with its role in hunger signaling to the brain and leads to excess food intake. Increased circulating leptin production is additionally associated with insulin resistance, infertility, and reproductive success (Mahmood et al., 2013).

Overall, excess fat accumulation leads to increased adipokines and secretion of pro-inflammatory adipocytokines, directly contributing to a low grade, chronic inflammatory environment (Heymsfield et al., 2017). This differs from typical inflammation because there are no signs of acute inflammation, yet cardiovascular and metabolic disorders are still developed through the same mechanisms.

The relationship between obesity and excess fat accumulation leads to the development of increased body fat, diabetes, and high blood pressure. The combination of these health conditions have chronic implications. Metabolic syndrome is more likely to develop, characterized by long term and potentially fatal outcomes such as congestive heart failure, stroke and chronic kidney disease. This is prevalent in one third of the obese population (Mahmood et al., 2013). Other common diseases include obstruction of air pathways, sleep apnea, osteoarthritis, and an increase in psychiatric disorders (Heymsfield et al., 2017). In addition, up to $47.6 \%$ of coronavirus disease 19 (COVID-19) is associated with obesity, with a higher risk of developing severe symptoms and complications (Simonnet et al., 2020). These have been shown to be independent of other illnesses, such as cardiovascular disease (Stefan et al., 2020). 


\section{Maternal Obesity}

Epidemiological studies indicate that a large proportion of women that are within reproductive age are obese, and these trends will only become more abundant with time. In 2017-2018, women in the United States between the age of 20-39 were reported to have a 39.9\% obesity rate, and were more prone to severe obesity compared to men (Hales et al., 2020). Additionally, projections show that by 2030 , the increase in obesity nationwide will mostly affect women, black non-Hispanic, and low income adults. Severe obesity will be the most common BMI category for each of these groups within the next ten years (Ward et al., 2020).

Obesity is shown to be a leading factor in developing chronic disease, and is proven to have long term health consequences. Considering this, it is important to understand how obesity impacts reproductive processes. This includes both maternal and fetal complications.

\section{Maternal Risks}

Maternal health is compromised in the setting of obesity, as obese mothers are at increased risk of excessive weight gain during pregnancy, which further affects their health conditions. Pregnant obese women have a 4-9 times greater chance of developing gestational diabetes, hypertension and preeclampsia (Poston et al., 2016). Preeclampsia especially has severe long term consequences, causing damage to vital organs such as the cardiovascular system, kidneys and liver. There is also a greater risk of complications during labor and delivery. Higher BMI directly corresponds to complications with conception, induced labor, miscarriages, cesarean section, and risk for postpartum hemorrhages. Post labor, healing times and complications with open wounds are significantly increased. Lastly, there are long term risks in obese pregnancies, as long term weight retention poses health risks as well as complications for future pregnancies (Poston et at., 2016).

\section{Fetal Risks}

Regardless of successful conception, fetal and infant survivals are at risk. Offspring are commonly delivered large for gestational age, predisposing them to develop disease in their adult 
life (Posten et al., 2016). These risks exhibit sexual dysmorphism, where male offspring are more likely to experience poor developmental outcomes (Myatt et al., 2016). The risk for mortality increases, with increased risk for unexplained stillbirth. In the first year of life, increased death rates also correlate to the BMI of the mother. Intergenerationally, the rate of heritability for BMI can range between $40 \%$ to $70 \%$ (Heymsfield et al., 2017). Offspring have a higher risk for high adiposity, waist circumference, leptin, and higher systolic blood pressure (Mahmood et al., 2013). Studies have indicated that gestational diabetes mellitus (GDM) puts offspring at risk for developing diabetes themselves. In these studies, offspring born to pre-diabetic or non-diabetic mothers had a much lower risk of diabetes compared to diabetic mothers. After GDM develops during the pregnancy, the risk for future diabetes significantly increases and correlates with the hyperglycemic intrauterine environment in which the fetus develops (Mahmood et al., 2013). Additionally, there is a significant increase in the risk of congenital anomalies for offspring of obese mothers including defects of the brain and spinal cord, excess cerebrospinal fluid, health and limb defects, and cleft palate (Poston et al., 2016). Overall, babies born to obese mothers are more likely to develop diabetes, heart disease, hypertension, and premature mortality later in life (Mahmood et al., 2013).

In addition to physical complications, there is an increased risk for the development of psychiatric and learning disabilities. Both maternal obesity and gestational diabetes contribute to increased risk of autism spectrum disorder and learning disabilities of offspring (Li et al., 2016). There is a 1 to 3-fold increased risk in these cases (Edlow et al., 2016). Offspring are also susceptible to developing other disorders, such as cerebral palsy, anxiety, depression, schizophrenia, and eating disorders. Considering maternal obesity is directly linked to long term abnormalities for offspring, it is important to understand the long term implications of this.

\section{Developmental Programming}

Studies indicate that lifestyle and genetics contribute to obesity and metabolic disease, and also originate from the prenatal, fetal, and postnatal environment (Taylor et al., 2007). Thus, these altered environments during the early stages of development are thought to be the reason behind the increased risk for chronic disease much later in life. The theory that links altered 
environments and the disruption of growth, development, and adult disease is known as Developmental Programming. This theory was initially introduced by David Barker, who theorized that these risks do not stem from genetic factors, but instead from early environments that physically "program" offspring for disease (Olsen, 2014).

Barker focused on the role of nutrition and its relationship to epigenetic modifications. Specifically, his studies looked at offspring of low-income neighborhoods, who later in life developed a very high risk for mortality, type 2 diabetes, cardiovascular disease, stroke, lung disease, and polycystic ovary syndrome (Padmanabhan et al., 2016). Initially, Barker's hypothesis was linked to undernutrition and low birth weight, where insulin resistance, reduction in growth, and disruption in metabolic processes occurred. Barker showed that undernutrition causes an adaptive response to ensure fetal survival by centering energy and growth towards vital organs such as the heart and brain (Olsen, 2014). This is known as a "thrifty" phenotype, as this reduction of energy towards other organs increases their vulnerability later in life. This is because with age, the ability for plasticity decreases, and these phenotypes become fixed and compromises overall function (Padmanabhan et al., 2016). Ultimately, Barker demonstrated how undernutrition plays a large role in developmental programming of offspring directly in response to their environment.

\section{Programming Effect of Maternal Overnutrition: Activation of Disease}

Considering the support regarding how undernutrition drives developmental programming, these theories are also considered for overnutrition, as the prevalence of obesity continues to rise within our community. More recent findings are geared towards a "2-hit" hypothesis, explaining the exposure to multiple risk factors within different stages of life ultimately program a maladaptive response. These factors are considered to be "stressors," where during perinatal life, a "first-hit" may be caused by genetic susceptibility and an altered fetal environment. During postnatal life, environmental stressors activate a "second-hit" in which underlying disease may be activated (Padmanabhan et al., 2016).

There are multiple sources of "stressors" which can activate disease during perinatal and postnatal development. As previously discussed, obesity is associated with decreased fertility 
and increased risk of medical intervention. The use of medical intervention in conception can be considered a perinatal stressor, and has been shown to cause epigenetic changes in the offspring (Padmanabhan et al., 2016). Additional stressors include lifestyle habits such as physical activity, diet, and substance abuse, with up to $16 \%$ of pregnant women using drugs within the U.S. The effect of these stressors is clearly demonstrated between siblings, where weight gain between pregnancies causes the development of metabolic disease for the second child, while the first is metabolically not compromised (Padmanabhan et al., 2016). Programming through these stressors may be due to maternal overnutrition and stress, which increase the risk for developing disease (Padmanabhan et al., 2016). A second stressor includes development of offspring during postnatal life, where exercise, nutrition, and lifestyle choices can also induce the activation of underlying disease.

Further stressors such as endocrine disrupting chemicals (EDCs), found within beauty and health supplies, contribute to perinatal and postnatal development. They interfere with metabolic and hormonal processes, and alter the secretion, synthesis, and binding of many molecular factors. EDCs have been shown to be transferred to offspring via lactation, and are linked to obesity, cardiovascular disease, type 2 diabetes, and cancer (Padmanabhan et al., 2016). The combination of these various perinatal and postnatal stressors are hypothesized to activate chronic disease in offspring.

\section{Epigenetic Programming}

The altered physical state of obesity changes the way in which other molecular and physiological mechanisms utilize and expedite energy, and how metabolic processes occur. Obesity is associated with epigenetic modifications of proteins, enzymes, and DNA. This includes alterations of DNA methylation, packing, and expression, which interfere with glucose metabolism (Padmanabhan et al., 2016). Oxidative stress is upregulated in obese individuals, which damages proteins and DNA due to the overexpression of catalytic enzymes. This is a potential source for programming of obesity and diabetes in offspring (Padmanabhan et al., 2016). 


\section{Hyperlipidemia and Programming}

Current literature has demonstrated that the environment of maternal obesity chronically programs offspring for metabolic disease, although few have directly indicated the long term consequences of this. Generally, these studies have demonstrated that overnutrition during pregnancy and lactation period are correlated to obesity of the offspring (Tayor et al., 2007). The exact mechanisms in which this occurs remain understudied, although hyperlipidemia is thought to be a major source for this programming to occur.

During early stages of pregnancy, increased levels of insulin lead to excess storage of lipids and upregulation of lipid transport proteins. Placental tissue samples of obese mothers are marked by increased lipid markers, where excess adipose accumulation leads to increased fatty acid delivery to the fetus through the placenta (Heerwagen, Margaret J. R., et al., 2010). Perinatal exposure to hyperlipidemia has been demonstrated to cause irreversible adipose levels and alter organ development through the activation of pro-inflammatory pathways, mitochondrial function, stem-cell development, metabolism, and interference with transcriptional processes of genes relating to lipid production (Heerwagen, Margaret J. R., et al., 2010).

Postnatal feeding also contributes to offspring hyperlipidemia. Animal models have demonstrated that the suckling period contributes to high levels of adiposity in offspring. In these cases, mothers consuming a high fat diet during the suckling period is correlated to hyperlipidemia within offspring. Post-suckling, if offspring are fed a normal diet, signs of adiposity and hypertension within adulthood remain present, which signifies these characteristics are irreversible This phenotype can then be passed down to the second generation, indicating that these characteristics are somehow heritable (Taylor et al., 2007).

Hyperlipidemia and high caloric diets target the central nervous system, where appetite and hunger suppression of offspring are altered through hypothalamic programming (Taylor et al., 2007). Maturation of the hypothalamus occurs during the gestation period and through early postnatal life, thus, the hypercaloric and hyperlipidemic environment is thought to alter these hunger related mechanisms throughout the pregnancy and suckling period by the transfer of fat across the placenta and breast milk (Taylor et al., 2007). Studies have indicated that overfeeding 
of rats and sheep in utero and postnatally is correlated to excess appetite and fat accumulation on a hypothalamic level (Taylor et al., 2007).

\section{Conclusions}

Maternal obesity has been clearly demonstrated to be linked to developmental programming of offspring. The environment of the gestational period alone is said to directly program for chronic disease and fetal risks. Despite this strong correlation, the actual mechanisms in which this programming occurs remain poorly understood. Potential sources include hyperlipidemia interference with cell signaling pathways and gene expression. Further research should be conducted in order to understand the etiology of these mechanisms, as maternal obesity continues to be a prevalent health issue for our community. 


\section{Works Cited}

Aiken, Catherine E., and Susan E. Ozanne. "Transgenerational Developmental Programming." Human Reproduction Update, vol. 20, no. 1, 2013, pp. 63-75., doi:10.1093/humupd/dmt043.

Alfaradhi, Maria Z., and Susan E. Ozanne. "Developmental Programming in Response to Maternal Overnutrition.” Frontiers in Genetics, vol. 2, June 2011, doi:10.3389/fgene.2011.00027.

Burton, Graham J. The Placenta and Human Developmental Programming. Cambridge University Press, 2011.

Castro, A.m., et al. "Low-Grade Inflammation and Its Relation to Obesity and Chronic Degenerative Diseases." Revista Médica Del Hospital General De México, vol. 80, no. 2, 2017, pp. 101-105., doi:10.1016/j.hgmx.2016.06.011.ps

Challier, J.c., et al. “Obesity in Pregnancy Stimulates Macrophage Accumulation and Inflammation in the Placenta.” Placenta, vol. 29, no. 3, 2008, pp. 274-281., doi:10.1016/j.placenta.2007.12.010.

Chen, Linlin et al. "Inflammatory responses and inflammation-associated diseases in organs." Oncotarget vol. 9,6 7204-7218. 14 Dec. 2017, doi:10.18632/oncotarget.23208

Edlow, Andrea G. "Maternal Obesity and Neurodevelopmental and Psychiatric Disorders in Offspring." Prenatal Diagnosis, vol. 37, no. 1, July 2016, pp. 95-110., doi:10.1002/pd.4932.

Fontana, L., et al. "Visceral Fat Adipokine Secretion Is Associated With Systemic Inflammation in Obese Humans.” Diabetes, vol. 56, no. 4, July 2007, pp. 1010-1013., doi:10.2337/db06-1656. 
Furukawa, Shigetada, et al. "Increased Oxidative Stress in Obesity and Its Impact on Metabolic Syndrome.” Journal of Clinical Investigation, vol. 114, no. 12, 2004, pp. 1752-1761., doi:10.1172/jci21625.

Hales, Craig M., et al. "Prevalence of Obesity and Severe Obesity Among Adults: United States, 2017-2018." NCHS Data Brief, no. 360, Feb. 2020.

Heerwagen, Margaret J. R., et al. "Maternal Obesity and Fetal Metabolic Programming: a Fertile Epigenetic Soil.” American Journal of Physiology-Regulatory, Integrative and Comparative Physiology, vol. 299, no. 3, 2010, doi:10.1152/ajpregu.00310.2010.

Heymsfield, Steven B., and Thomas A. Wadden. "Mechanisms, Pathophysiology, and Management of Obesity." The New England Journal of Medicine, Jan. 2017, pp. 254-266., doi:10.1056/NEJMra1514009.

Li, M., et al. "The Association of Maternal Obesity and Diabetes With Autism and Other Developmental Disabilities.” Pediatrics, vol. 137, no. 2, 29 Feb. 2016, doi:10.1542/peds.2015-2206.

Mahmood, Tahir, and Sabaratnam Arulkumaran. Obesity: a Ticking Time Bomb for Reproductive Health. Elsevier, 2013.

Myatt, Leslie and Alina Maloyan "Obesity and Placental Function.” Seminars in Reproductive Medicine, vol. 34, no. 01, 2016, pp. 042-049., doi:10.1055/s-0035-1570027.

Olsen, Jørn. “David Barker (1938-2013) - a Giant in Reproductive Epidemiology.” Acta Obstetricia Et Gynecologica Scandinavica, vol. 93, no. 11, 2014, pp. 1077-1080., doi:10.1111/aogs.12378.

Padmanabhan, Vasantha, et al. "Developmental Programming, a Pathway to Disease." Endocrinology, vol. 157, no. 4, 2016, pp. 1328-1340., doi:10.1210/en.2016-1003. 
Poston, Lucilla, et al. "Preconceptional and Maternal Obesity: Epidemiology and Health Consequences." The Lancet Diabetes \& Endocrinology, vol. 4, no. 12, 12 Oct. 2016, pp. 1025-1036., doi:10.1016/s2213-8587(16)30217-0.

Simonnet, Arthur, et al. "High Prevalence of Obesity in Severe Acute Respiratory Syndrome Coronavirus-2 (SARS-CoV-2) Requiring Invasive Mechanical Ventilation.” Obesity, 2020, doi:10.1002/oby.22831.

Stefan, Norbert, et al. "Obesity and Impaired Metabolic Health in Patients with COVID-19." Nature Reviews Endocrinology, 2020, doi:10.1038/s41574-020-0364-6.

Taylor, P. D., and L. Poston. "Developmental Programming of Obesity in Mammals." Experimental Physiology, vol. 92, no. 2, Jan. 2007, pp. 287-298., doi:10.1113/expphysiol.2005.032854.

Warner, Matthew J., and Susan E. Ozanne. "Mechanisms Involved in the Developmental Programming of Adulthood Disease." Biochemical Journal, vol. 427, no. 3, 2010, pp. 333-347., doi:10.1042/bj20091861

Youfa Wang, May A Beydoun, Jungwon Min, Hong Xue, Leonard A Kaminsky, Lawrence J Cheskin, "Has the prevalence of overweight, obesity and central obesity levelled off in the United States? Trends, patterns, disparities, and future projections for the obesity epidemic”, International Journal of Epidemiology, dyz273, https://doi.org/10.1093/ije/dyz273

Ward, Zachary J., et al. "Projected U.S. State-Level Prevalence of Adult Obesity and Severe Obesity." New England Journal of Medicine, vol. 381, no. 25, 2019, pp. 2440-2450., doi:10.1056/nejmsa1909301. 